\title{
Utilização de Redes Bayesianas para Avaliação da Qualidade de Experiência do Usuário: Um Estudo de Caso Com Segunda Tela
}

\author{
Joahannes Bruno Dias da Costa $^{1}$, Marcos Cesar da Rocha Seruffo ${ }^{1}$, \\ Marianne Kogut Eliasquevici ${ }^{2}$, Daniel da Silva Souza ${ }^{1}$ \\ ${ }^{1}$ Faculdade de Computação (FACOMP) - Universidade Federal do Pará (UFPA) \\ 68746-360 - Castanhal - PA - Brasil \\ ${ }^{2}$ Instituto de Ciências Exatas e Naturais (ICEN) - Universidade Federal do Pará (UFPA) \\ 66075-110 - Belém - PA - Brasil \\ \{joahannes, seruffo, marianne, danielssouza\}@ufpa.br
}

\begin{abstract}
This article presents a study on evaluation of the quality of the user experience from the use of a second screen application that interacts with television programming. For this, it was employed a model of computational intelligence - Bayesian network - to correlate the main factors that influence the user experience, these being: human factors, and system context. As a result, a discussion that aims to promote the improvement of user experience through criteria to assess applications, either during the design phase or during its operation.
\end{abstract}

Resumo. Este artigo apresenta um estudo sobre avaliação da qualidade da experiência do usuário a partir do uso de um aplicativo de segunda tela que interage com a programação televisiva. Para isto, foi empregado um modelo de inteligência computacional - Rede Bayesiana - para correlacionar os principais fatores que influenciam na experiência do usuário, sendo estes: fatores humanos, sistema e de contexto. Como resultado, é apresentada uma discussão que visa promover a melhoria da experiência do usuário por meio de critérios que permitam avaliar os aplicativos, quer seja durante a sua fase de design ou durante sua fase de operação.

\section{Introdução}

O crescimento do uso de múltiplas telas no momento da assistência à televisão (TV), no Brasil e no mundo, tem aberto perspectivas instigantes para os desenvolvedores interessados em entrar no mercado de segunda tela. A segunda tela nada mais é do que um recurso adicional para a primeira tela, tais como um notebook, tablet ou smartphone com um sistema operacional capaz de executar aplicativos instalados [Soskic 2014].

Para que além de os telespectadores simplesmente sentarem e assistirem o conteúdo televisivo, agora possuem a oportunidade de participar, comentar, escolher e compartilhar o que estão assistindo. Um fator que colabora para estas novas possibilidades é o barateamento do preço dos dispositivos móveis, aliado ao aumento do poder de processamento que os mesmos estão ganhando. 
São várias as emissoras que estimulam a interação entre o telespectador e o programa televisivo por meio das redes sociais, fazendo com que esse mercado venha crescendo exponencialmente. Uma pesquisa indica que 57\% dos brasileiros usam o Facebook ao mesmo tempo em que assistem TV. Dentre eles, 39\% escolhem um dispositivo móvel como sua segunda tela. Nos horários entre 20h00min e 00h00min, oito em cada dez brasileiros usam a rede social enquanto estão vendo TV, representando $81 \%$ dos telespectadores [IpsosMediact 2014].

Durante a Copa do Mundo de 2014, por exemplo, enquanto os jogos eram transmitidos, os telespectadores teciam comentários nas redes socais. No Twitter foram registrados 672 milhões de tweets sobre o Mundial, transformando o torneio no evento mais tuitado de todos os tempos; já no Facebook foram registradas três bilhões de interações entre usuários desde o início do evento, sendo a primeira vez que essa marca é atingida [Copa 2014].

Preocupadas em não perder mercado, emissoras brasileiras (Rede Globo, Rede Record, TV Cultura e o Sistema Brasileiro de Televisão - SBT) começam a se mobilizar dentro deste segmento para a TV aberta e lançam aplicativos para que os telespectadores acompanhassem e interagissem com a programação que estava sendo exibida. Dentre algumas dessas experiências de segunda tela no Brasil, se encontram: o aplicativo desenvolvido para o reality show Menino de Ouro, exibido pelo SBT e o reality show Superstar, exibido pela Rede Globo.

Toda essa transformação tem impacto na qualidade de experiência do usuário, o que implica na necessidade de se investigar de forma mais profunda a forma com que o usuário se comporta e interage com aplicações, ou mesmo o porquê do mesmo não ter interesse em interagir, para que se possa prover serviços que satisfaçam os diversos perfis de usuários existentes.

Diante deste contexto, a contribuição deste artigo é a proposição de um estudo sobre a qualidade da experiência do usuário no momento da interação ${ }^{1}$ com um aplicativo de segunda tela, empregando a técnica de inteligência computacional Rede Bayesiana (RB) para correlação dos dados aferidos. Por meio da extração de conhecimento da base de dados obtida durante os testes realizados com o aplicativo, torna-se possível a montagem de um arcabouço que identifica fatores que avaliam o uso de aplicativos de segunda tela, quer seja durante a sua fase de design ou operação.

O restante do trabalho está organizado da seguinte forma: a Seção 2 apresenta a fundamentação teórica aplicada com conceitos de qualidade de experiência e sobre Redes Bayesianas; a Seção 3 mostra alguns trabalhos correlacionados que tratam de temas presentes nesse trabalho; a Seção 4 explicita o estudo de caso, com a arquitetura montada para avaliação, o aplicativo desenvolvido, o cenário de testes e os dados extraídos para análise; a Seção 5 apresenta os resultados e discussões da aplicação da metodologia proposta; e por fim, a Seção 6, apresentada conclusões sobre a pesquisa apresentada e apontamentos para trabalhos futuros.

\footnotetext{
${ }^{1}$ Entende-se como interação qualquer ação que o usuário realiza sobre o dispositivo de segunda tela, que nesse caso, é no smartphone ou tablet que está com o aplicativo instalado.
} 


\section{Fundamentação Teórica}

\subsection{Qualidade de Experiência}

Um conceito que tem sido muito empregado para avaliar serviços computacionais é a Qualidade da Experiência (QoE) que, para a International Telecommunication Union [ITU-T 2007], é a aceitabilidade geral de um aplicativo ou serviço, conforme percebida subjetivamente pelo usuário final, incluindo os efeitos de sistema (qualidade da rede, serviços de infraestrutura, entre outros).

No geral, aceitabilidade pode ser influenciada pelo contexto e expectativas do usuário [ETSI 2009]. Conforme [Geerts et al. 2010], as expectativas dos usuários, a satisfação e a QoE (percebida) estão sendo reconhecidas como determinantes para o sucesso de qualquer tecnologia, sendo ainda mais importante do que o desempenho isolado tecnológico e de excelência, em muitos casos definido como Qualidade de Serviço (QoS).

A QoE tem como intenção mapear os fatores que exercem influência na experiência de uso da tecnologia. A influência pode vir de qualquer característica de um usuário, sistema, serviço, aplicativo ou contexto. Assim, a literatura classifica QoE como uma interseção de fatores de influência, ou seja: Fatores Humanos (FH), Fatores de Contexto (FC) e Fatores de Sistema (FS).

\subsection{Redes Bayesianas}

A utilização de modelos de inteligência computacional na resolução de problemas é muito comum e eficiente, pois é sabido o alto nível de confiabilidade que os mesmos possuem. Neste estudo, foi utilizada a técnica de Rede Bayesiana (RB), que pode ser definida como uma rede probabilística constituída por uma estrutura gráfica e uma distribuição de probabilidades a ela associada [Sarabando 2011]. Sua representação utiliza grafos acíclicos direcionados com suas respectivas probabilidades condicionais.

Os eventos que influenciam uma variável influenciam diretamente a quantidade de informação na outra variável devido à correlação existente entre elas [Ferrari 2012]. Para que se possa obter essa correlação entre variáveis é preciso relacionar as suas probabilidades, e tal conexão é obtida a partir da aplicação do teorema de Bayes, base das RBs, já que ele investiga a ocorrência de novas probabilidades com base em outras existentes.

Como a quantização do grau de influência que uma variável tem sobre as outras é a base para medir a QoE, se buscou uma forma de medição dessa influência e foi então que se chegou ao Índice de Carga, ou Entropia Condicional, das variáveis. Sendo assim, quando as variáveis $x$ e $y$ estão correlacionadas, isto é, então, a entropia de cada uma das variáveis é condicionada a outra e isso mostra o quanto uma variável influencia na outra.

\section{Trabalhos Correlacionados}

Diante do contexto de grande utilização de diversos dispositivos juntamente com a TV, estudos vem surgindo para investigar de que forma tais serviços podem ser melhor ofertados para os usuários.

Dentre os autores que apoiam a ideia de considerar, não somente dados ligados ao sistema em si, como também fatores ligados ao ser humano (emoções, experiência prévia, etc.) ao se avaliar a experiência de uso de determinado sistema pode-se citar 
[Piccolo et al. 2010], que apresenta uma metodologia para avaliação da QoE de serviços de TV interativa que considera tanto fatores subjetivos relacionados ao comportamento humano, quanto fatores objetivos referentes ao desempenho do serviço oferecido. E também [Yong 2013] que discute a elaboração de um quadro conceitual para a experiência do usuário com o objetivo de medir a resposta emocional, comportamental e reflexiva de quem utiliza determinado sistema.

O grau de satisfação do usuário em relação a um determinado serviço é crucial para o estreitamento das operadoras de serviços e os usuários. O trabalho de [Zhou 2013] descreve um estudo que comprova a importância de se avaliar serviços de TV móvel com base no usuário. A pesquisa identifica que pontos como a facilidade de utilização, a velocidade de acesso e a qualidade do conteúdo têm efeitos significativos sobre a experiência de utilização desses serviços. Esse tipo de estudo pode ser um forte aliado para análise mercadológica, em que se busca traçar o perfil do usuário e entregar um serviço direcionado.

Referente ao emprego de Redes Bayesianas, como técnica de inteligência computacional de aplicação eficaz em diversos domínios, que não necessariamente o de QoE. A pesquisa de [Xenos 2004], por meio da aplicação de RB, avalia o comportamento de estudantes de um curso de computação ofertado na modalidade à distância, para verificar o quanto os fatores presentes nesse cenário influenciam no rendimento acadêmico dos estudantes e prevê possíveis egressos com base em previsões que esse tipo modelo oferece. E também [Da et al. 2011] que emprega RB com o objetivo de criar um modelo de escolha de recursos de aprendizagem, de acordo com as características pessoais dos alunos, utilizando feedback dos resultados dos exames dos cursos de formação como amostras de dados.

O trabalho de [Zhu et al. 2015] reforça a ótica de avaliação da QoE levando em consideração não apenas as propriedades técnicas multimídias do serviço oferecido, mas também o contexto social e fatores ligados ao ser humano para que essa avaliação seja mais completa e que estime suficientemente a satisfação do usuário final. Isso nos motivou na construção deste estudo que busca a criação de uma metodologia de avaliação da QoE que considera fatores ligados ao sistema, fatores humanos e fatores ligados ao contexto em que esse usuário está inserido. Este trabalho serviu de base para o desenvolvimento deste artigo e ressaltou a importância de estudos desta natureza.

Conforme exposto, são vários os estudos sobre QoE e inteligência computacional que podem ser encontrados na literatura. Dentro deste enfoque, este artigo traz inovações por conseguir relacionar, em um mesmo trabalho, características não encontradas, ou encontradas de forma dispersa na literatura pesquisada para apresentar a construção de um modelo genérico para avaliação de QoE que leva em consideração tanto parâmetros de sistema, obtidos de forma dinâmica da aplicação à medida em que o usuário interage com a aplicação, como também parâmetros extraídos do preenchimento de questionários, que englobam fatores humanos e de contexto.

\section{Estudo de Caso}

\subsection{Arquitetura Montada}

Foi montada uma arquitetura, mostrada na Figura 1, para o estudo de caso que descreve a abordagem metodológica empregada, envolvendo desde a etapa dos testes com usuários 
até o uso de RB para modelar a QoE de um aplicativo de segunda tela, tendo como base os fatores constantes da QoE.

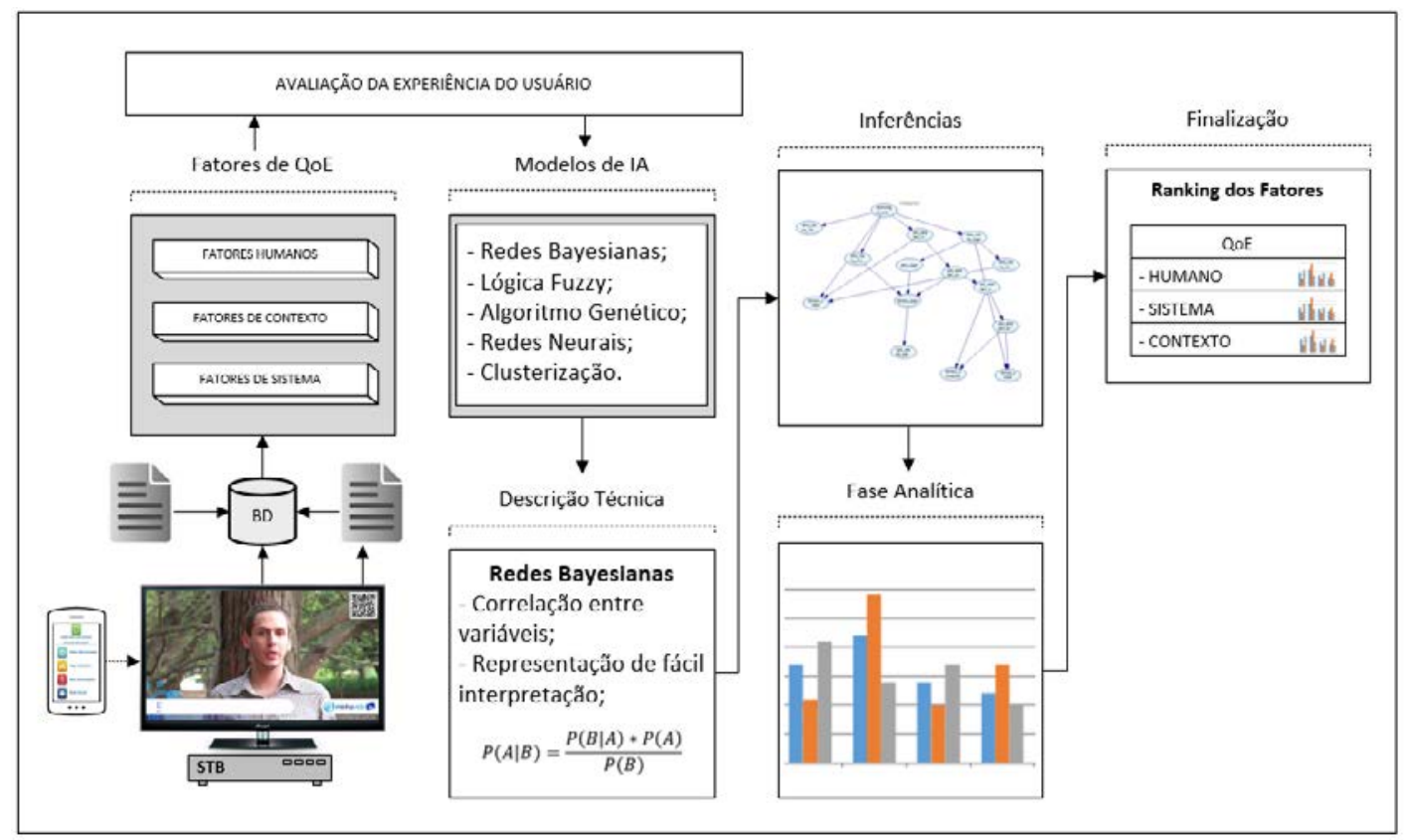

Figura 1. Arquitetura montada para testes e extração de conhecimento

Um programa televisivo foi exibido e o usuário podia interagir através de uma segunda tela (smartphone, por exemplo). Todo processo de interação dos usuários com o aplicativo DFapp foi monitorado e, ao final, foram aplicados instrumentos (questionários) avaliativos, para que se pudesse obter uma base de dados contendo o perfil do usuário e seus dados de interação, representado, assim, a experiência do usuário. A partir desta etapa, foram aplicados diferentes modelos de inteligência computacional, visando estabelecer qual mais se adequava ao objetivo proposto neste estudo e optou-se pela Rede Bayesiana como técnica de inteligência computacional a ser utilizada para a predição de resultados.

Tendo por base a modelagem das redes, inferências foram realizadas para obtenção do grau de correlação entre as variáveis (fatores) presentes, permitindo a visualização das tabelas de probabilidades de cada correlação observada. Por fim, foi feita uma análise para identificar o quanto determinado fator exerce de influência na experiência de uso do aplicativo de segunda tela, elencando os fatores envolvidos no sistema proposto.

\subsection{Aplicativo Utilizado}

O aplicativo Dengue Fever App (DFapp) foi desenvolvido por pesquisadores do Laboratório de Desenvolvimento de Sistemas (LADES), da Faculdade de Computação (FACOMP), do Campus de Castanhal da UFPA. Voltado para área de saúde ( $t$-health), o DFapp permite levar informações sobre a Dengue, uma doença tropical com índices crescentes de ocorrência em algumas regiões do Brasil.

Na Figura 2 estão ilustradas: a tela inicial do aplicativo após a sincronização; (a tela com o vídeo que está sendo exibido no smartphone (segunda tela), de forma sin- 

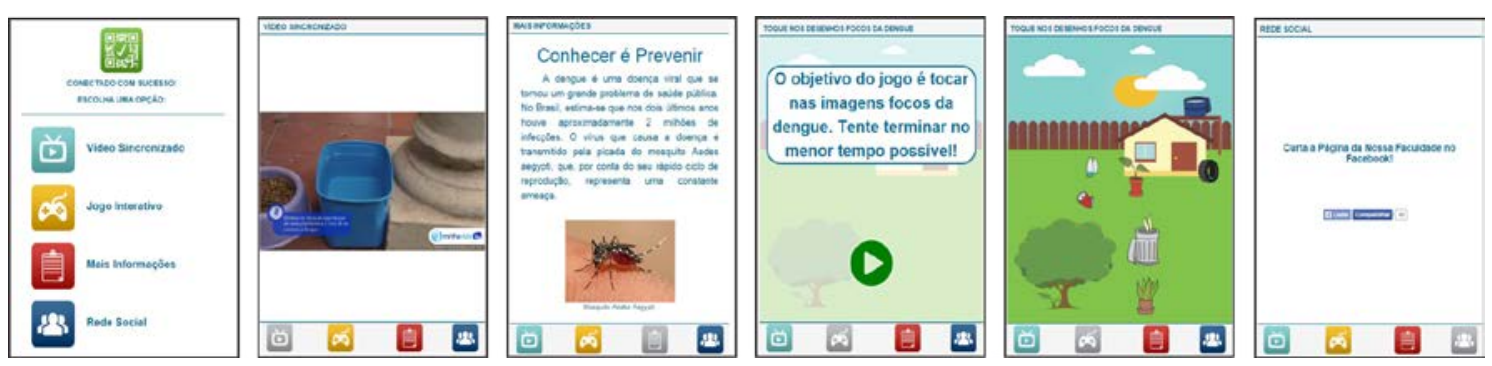

Figura 2. Telas do aplicativo de segunda tela - DFApp

cronizada ao que está sendo transmitido na televisão (primeira tela); e uma tela com conteúdo extra que fornece aos usuários mais informações sobre a Dengue. As outras opções do aplicativo: um jogo interativo em que o usuário tem que identificar e eliminar os focos da doença, tendo o tempo para esta ação cronometrado e exibido ao final da eliminação de todos os focos; e a possibilidade de o usuário acessar o Facebook e compartilhar informações sobre o aplicativo e sobre a doença [Seruffo et al. 2015], respectivamente.

A temática do aplicativo desenvolvido é da área da saúde que possui uma função social de levar informação a respeito da doença da Dengue à população. Caso o aplicativo desenvolvido fosse centrado em outra temática, os resultados apresentados poderiam ter sido diferentes. Isso significa dizer que analisar o conteúdo de uma aplicação é tão fundamental quanto os outros fatores estudados.

\subsection{Definição do Cenário e Protocolo de Testes}

O cenário montado para os testes foi composto por um dispositivo móvel e um aparelho de TV, ambos acessando uma rede sem fio por meio de um Ponto de Acesso conectado à Internet. Um servidor para representar um provedor de TV, responsável pela transmissão do vídeo online também fez parte do cenário. Os testes em laboratório foram realizados em ambiente controlado para facilitar a captura de informações. Durante um período de uma semana, se contou com a presença de 73 usuários, quantidade esta baseada em literaturas como [Norman 2004] e [Faulkner 2003]. É importante ressaltar que os testes foram concebidos a partir da perspectiva tanto qualitativa quanto quantitativa.

As idades variaram entre a faixa etária de 13 a 70 anos, sendo de diversas classes sociais e níveis de escolaridade, gerando assim uma base de dados consolidada e diversificada.

Visando sistematizar a bateria de testes, foi criado um protocolo que pudesse ser seguido pelos pesquisadores envolvidos. O processo de captura das métricas subjetivas, concernentes a cada usuário, tal como proposto em [ITU-T 2007], [Geerts et al. 2010] e [Menkovski et al. 2011]. Primeiro o usuário era submetido a um questionário de dados pessoais $^{2}$, o qual deveria ser preenchido de forma orientada, visando traçar um perfil socioeconômico. Este questionário foi armazenado com as informações preliminares de cada participante.

Após, o usuário era encaminhado à outra sala, chamada de sala de interação, na qual recebia uma explicação básica sobre o teste e quais os objetivos do projeto de pes-

\footnotetext{
${ }^{2}$ Questionário 01 - Disponível em http://www.facompcastanhal.ufpa.br/lades/pesquisa/quest-dfapp.pdf
} 
quisa. A ideia foi ambientar os usuários antes de iniciar a utilização do aplicativo, contudo, não foi ensinado o uso da aplicação, deixando o usuário livre para experiência.

A partir daí a programação televisiva iniciava, sendo que a tela inicial de apresentação do aplicativo na TV exibia as seguintes mensagens: "A programação a seguir possui interatividade com segunda tela" e "Sincronize seu smartphone pelo QR Code que estará disponível". Na tela subsequente, o usuário era convidado a sincronizar o smartphone com a TV pela leitura do QR Code através da seguinte mensagem: "Para continuar, sincronize com a TV utilizando o QR Code do smartphone". Assim que o aplicativo era iniciado, o usuário poderia utilizar, de forma livre, as tecnologias disponíveis para os testes, supervisionado por uma pessoa da equipe do Laboratório. Estas mensagens foram inseridas na segunda versão do aplicativo, por ser constatado que mesmo com as explicações, poucos usuários entendiam a necessidade de sincronismo.

Ao final do teste de interação, o usuário era encaminhado para outra sala, para realizar uma entrevista em profundidade, norteada por perguntas contidas em um formulário previamente preparado ${ }^{3}$. $\mathrm{O}$ objetivo foi obter informações sobre: o experimento exercido pelo usuário; a facilidade da interação; a compreensão dos elementos das telas da aplicação nos diferentes dispositivos, TV e dispositivo móvel; o conhecimento prévio sobre aplicativos interativos; tempo de uso diário da TV e do serviço de Internet; e quais dispositivos mais usados junto com a TV, dentres outras questões, gerando uma base de dados sólida para o estudo ${ }^{4}$.

\subsection{Métricas Utilizadas}

Como métricas objetivas fez-se uso de 14 dados obtidos diretamente da utilização da aplicação (Tabela 1), tais como o tempo de interação do usuário no jogo, a quantidade de erros cometidos durante o uso da aplicação e durante o jogo mais especificamente, dentre outros, uma vez que a aplicação registra todos os dados das atividades realizadas pelo usuário. A definição dessas métricas foi baseada nos trabalhos correlatos apresentados na seção anterior.

Tabela 1. Variáveis do sistema

\begin{tabular}{|c|c|}
\hline $\begin{array}{l}\text { erro_icone_jogo } \\
\text { erro_jogo } \\
\text { erro_icone_social } \\
\text { erro_icone_video } \\
\text { qtd_selecao_conteudo } \\
\text { qtd_selecao_jogo } \\
\text { qtd_selecao_social }\end{array}$ & $\begin{array}{l}\text { qtd_selecao_video } \\
\text { tempo_conteudo } \\
\text { tempo_jogo } \\
\text { tempo_social } \\
\text { tempo_video } \\
\text { qtd_vezes_jogadas } \\
\text { erro_icone_conteudo }\end{array}$ \\
\hline
\end{tabular}

Ainda sobre a seção de testes, foi solicitado a cada usuário o preenchimento de outro questionário ${ }^{5}$ com 12 perguntas (variáveis) referentes à interface do aplicativo e sua experiência de uso. Cada pergunta continha uma escala com cinco alternativas possíveis (1 - Péssimo/Muito Difícil, 2 - Ruim/Difícil, 3 - Razoável/Mediano, 4 - Muito Bom/Muito Fácil e 5 - Excelente/Extremamente Fácil). A soma destas 12 variáveis com as 13 pro-

\footnotetext{
${ }^{3}$ Questionário 02 - disponível em: http://www.facompcastanhal.ufpa.br/lades/pesquisa/quest-dfapp.pdf

${ }^{4}$ Base de dados - disponível em: http://www.facompcastanhal.ufpa.br/lades/pesquisa/dados-dfapp.xlsx

${ }^{5}$ Questionário 03 - disponível em: http://www.facompcastanhal.ufpa.br/lades/pesquisa/quest-dfapp.pdf
} 
venientes do primeiro questionário, totalizaram as 25 métricas subjetivas (Tabela 2) que fizeram parte da modelagem das redes.

\begin{tabular}{ll} 
Tabela 2. Variáveis extraídas por questionários \\
\hline gênero & uso_paralelo_tv \\
conteudo_assistido & experiencia_uso_app \\
idade & interação_com_tv \\
escolaridade & interface_app \\
renda & memorizacao_interface \\
moram_com_vc & organizacao_infos_tv \\
tempo_uso_tv & organizacao_infos_celular \\
tipo_tv & comecar_app \\
costume_assistir_tv & sincronizacao_app_tv \\
uso_anterior_app & execucao_tarefas \\
tecnologia_acesso & tempo_adaptacao_app \\
uso_diario_tv & funcionalidade_app \\
uso_conjunto_tv & \\
\hline
\end{tabular}

A partir da definição das métricas, visando aplicar o modelo de RB para identificar qual fator é mais significativo na experiência do usuário ao interagir com o aplicativo, as 39 métricas foram classificadas, de acordo com a literatura [ITU-T 2007] e [Callet et al. 2012] em fator humano, fator de contexto e fator de sistema (Tabela 3).

Tabela 3. Variáveis usadas para fatores humanos, contexto e sistema

\begin{tabular}{cl}
\hline \multicolumn{1}{c}{ Fator } & \multicolumn{1}{c}{ Variáveis } \\
\hline Fator Humano & $\begin{array}{l}\text { Genero, faixa etária, experiência prévia, coletividade, conteúdo assistido, uso } \\
\text { anterior do app, experiência de uso do app, interação com a tv, interface do app, } \\
\text { memorização da interface do app e tempo de adaptação do app. } \\
\text { Tempo do vídeo, relações interpessoais, erros cometidos, uso paralelo, conteúdo } \\
\text { Fator de Contexto } \\
\text { assistido, renda, moram com você, tempo de uso da TV. } \\
\text { Tipo da tv, tecnologia de acesso, quantidade de seleção do vídeo, quantidade de } \\
\text { seleção jogo, quantidade de seleção do conteúdo, quantidade de seleção do social, } \\
\text { erro no ícone do vídeo, erro no ícone do jogo, erro no ícone do conteúdo, erro no } \\
\text { ícone do social, tempo no vídeo, tempo no jogo, tempo no conteúdo e tempo no } \\
\text { social. }\end{array}$ \\
\hline
\end{tabular}

\subsection{Resultados e Discussão}

Com as inferências realizadas por meio das RBs montadas e aplicando o processo de entropia condicional nas variáveis, pôde-se perceber, conforme Tabela 4, que o fator de Contexto exerce maior influência na experiência do usuário, seguido pelo Fator Humano e Fator de Sistema.

Tabela 4. Influência dos fatores no DFapp

\begin{tabular}{cc}
\hline Fator & Influência \\
\hline Contexto & $40 \%$ \\
Humano & $32 \%$ \\
Sistema & $28 \%$ \\
TOTAL & $\mathbf{1 0 0 \%}$ \\
\hline
\end{tabular}

De forma mais específica, a variação entre as probabilidades de ocorrências das alternativas do conjunto de variáveis no fator de contexto foi maior e mais significativa 
do que nos outros fatores. Por exemplo, em fator de contexto foram feitas 3 análises: erros cometidos, uso paralelo a tv e o costume de se assistir TV em conjunto, e dentre as três análises a que se mostrou mais impactante no contexto foi o uso paralelo da tv, pois de acordo com sua entropia condicional, influencia em $90 \%$ no contexto, ou seja, essa é a prática que mais influência na experiência do usuário, considerando apenas o contexto que o mesmo está inserido.

Ainda sobre o fator de contexto, usuários que não possuem acesso à Internet em suas residências, tendem a investir mais atenção no conteúdo transmitido na primeira tela (TV). Isto pode demonstrar quanto o processo de exclusão digital afeta a QoE, já que pessoas que não possuem acesso à Internet, ficam à margem do processo de apropriação de novas ferramentas que começam a se consolidar no mercado. Contudo, a partir do barateamento dos equipamentos eletrônicos, tais como smartphones, e a ampliação das tecnologias de acesso $3 \mathrm{G}$ e 4G, este cenário tende a sofrer alterações, gerando mais espaço para aplicações desta natureza.

Após análise das relações entre as variáveis, verificou-se que há uma tendência da assistência ao conteúdo televisivo de forma não coletiva. Entretanto, os usuários que assistem TV sozinhos passam menos tempo nesse tipo de atividade. Segundo [Zhu et al. 2015], "a co-visualização de vídeos com os amigos aumenta o nível de satisfação do usuário e aumenta a durabilidade da experiência”. Desta forma, os resultados alcançados neste trabalho, assim como os da literatura, apontam para uma maior satisfação por parte dos usuários quando assistem TV coletivamente.

Sobre os fatores de sistema, chama a atenção o fato de que os usuários que não possuem acesso à Internet em casa, passaram mais tempo na tela de conteúdo extra e cometem mais erros ao usar o aplicativo, este fato pode ser influenciado pois as pessoas ao interagirem com a aplicação, sentiram necessidade de usar a segunda tela para que obtivessem mais informações sobre o assunto, além disso, o elevado número de erros é ligado a falta de costume no uso de aplicações deste tipo, já que não há acesso à Internet. Por outro lado, pessoas com uma Internet de boa velocidade tendem a usar com frequência aplicações durante a assistência da TV, desta forma, essa experiência anterior refletiu nos testes, que apresentaram menos incidência de erros para esta categoria.

O sincronismo do vídeo da primeira com a segunda tela foi o recurso preferido dos usuários, que investiram mais tempo observando este funcionamento, isto é facilmente justificável pois trata-se de uma função ainda não disponibilizadas/disponíveis pelas emissoras de televisão do Brasil, portanto, gerando curiosidade do seu funcionamento pelos usuários, que ficaram surpresos sobre esta possibilidade de mobilidade.

De forma geral, pode-se extrair informações interessantes, como: qual gênero utiliza mais aplicativos e TV diariamente; o quanto as redes sociais influenciam e toma atenção dos indivíduos; a dificuldade que estudantes que não possuem Internet em suas casas têm ao utilizar aplicativos de segunda tela; o quanto utilizar diversos dispositivos ao assistir TV melhora o poder de percepção dos indivíduos, etc. Com as informações aqui apresentadas, o processo de análise do comportamento do usuário se torna mais fácil, já que o prisma de correlação entre as variáveis se mostrou mais claro e quantificável.

Outra observação importante que pôde ser realizada é o fato das mulheres que utilizaram o aplicativo erraram menos que os homens. Ou seja, tiveram mais facilidade 
no manuseio do aplicativo. Essa facilidade essa encontrada pode ser embasada pelo que ja foi exposto por [Charlton 2010], onde são apresentados dados que concluem a facilidade das mulheres para realização de determinadas atividades.

Dessa forma, ressalta-se que o fator que impacta mais intensamente na experiência do usuário é o de contexto. Sendo assim, o mundo ao redor do telespectador corrobora para a experiência de utilização que o mesmo tem. A carga de conhecimento que o usuário carrega, as suas relações interpessoais e o seu estado emocional em relação a determinado acontecimento são fundamentais neste processo de avaliação, conforme mostrado nos trabalhos correlatos. Considerar esses aspectos é de suma importância para os prestadores de serviços que tem como objetivo estarem sempre atrelados ao seu maior colaborador de feedbacks, que é o usuário final.

Salienta-se que estudos como este são fundamentais para a padronização de aplicativos que em sua fase de construção tendem atingir um público em específico. Vale ressaltar que o modelo aqui proposto não se restringe apenas a aplicativos de segunda tela, mas sim para qualquer tipo de aplicativo, já que esses podem gerar as mesmas informações de utilização que o DFapp gerou, contudo, para o estudo de caso apresentado, foram restritos os testes para segunda tela. Desta forma, o modelo é genérico e possui flexibilidade.

\section{Conclusão}

A evolução intrínseca da humanidade vem trazendo novas formas de se realizar tarefas, buscando sempre a comodidade de quem as realizam. A tecnologia vem para fortalecer essa evolução e proporciona velocidade importante nesse processo e atividades que antes eram simples e rotineiras hoje vem ganhando recursos que elevam a curiosidade e satisfação de uso.

Os modelos de avaliação da qualidade de experiência do usuário vêm tomando níveis altos de precisão e confiança. Com a utilização de técnicas computacionais que auxiliam nessa predição comportamental, tornam-se muito eficientes e precisos nas suas análises. Estudos recentes têm mostrado que a abordagem de considerar apenas parâmetros multimídia não pode suficientemente estimar a satisfação do usuário e que QoE depende de múltiplos fatores, além das propriedades técnicas de mídia. A contribuição deste trabalho está no ponto em que mescla fatores que antes não coexistiam, como, o quanto a percepção do usuário em relação à interface da aplicação é afetada dada sua condição financeira.

Esse trabalho contribui com a proposição de uma metodologia para avaliação da experiência do usuário para aplicativos utilizando como estudo de caso um aplicativo de segunda tela, mas não se restringe a avaliar apenas aplicativos desse tipo, traz um ganho para os desenvolvedores de aplicativos no que se refere a análise mercadológica de seus produtos e serviços, onde a preocupação que antes se direcionava apenas para qual tecnologia utilizar, hoje se mostra muito mais latente analisar o público que fará uso do produto final. Saber seus gostos, localização, bagagem de conhecimento e outros aspectos ligados ao contexto que esse usuário está inserido, como este estudo mostra, precisa de uma parcela maior de atenção na hora de se desenvolver um aplicativo.

O contexto que o usuário está inserido, bem como seu comportamento, traz informações importantíssimas para a definição do seu perfil como utilizador de certo sis- 
tema. Obter essa informação de forma clara e concisa traz benefícios para quem pretende lançar um aplicativo ou até mesmo melhorar um já existente. Pensando nisso, deve-se buscar formas para avaliar essa experiência que o usuário tem ao utilizar aplicativos e este trabalho propõe um novo método de obtenção dessas informações utilizando RB.

Se conclui que para uma análise mais fidedigna ao contexto geral de uso de um aplicativo, é crucial considerar fatores tanto ligados ao sistema (tecnologia de acesso, qualidade da transmissão de vídeo, etc.) e também fatores ligados ao ser humano que faz uso desse sistema (idade, genero, experiência prévia, etc).

Como trabalhos futuros pretende-se utilizar outros métodos de inteligência computacional para comparar os resultados obtidos, visando consolidar ainda mais a ideia proposta e mostrar um novo rumo que as fórmulas de avaliação podem tomar, considerando sempre os diversos fatores que influenciam na observação de um aplicativo ou serviço.

\section{Agradecimentos}

Os autores agradecem à Universidade Federal do Pará (UFPA). E também à Faculdade de Computação (FACOMP) e ao Laboratório de Desenvolvimento de Sistemas (LADES), ambos do campus de Castanhal, pelo apoio no desenvolvimento deste trabalho.

\section{Referências}

Callet, P. L., Möller, S., Perkis, A., et al. (2012). Qualinet white paper on definitions of quality of experience. European Network on Quality of Experience in Multimedia Systems and Services (COST Action IC 1003).

Charlton, G. (2010). Why are women so intelligent? the effect of maternal iq on childhood mortality may be a relevant evolutionary factor. Medical hypotheses, 74(3):401-402.

Copa (2014). Copa do mundo lidera estatísticas de 2014 do twitter e do facebook. http://www.copa2014.gov.br/pt-br/noticia/copa-do-mundo-lidera-estatisticasde-2014-do-twitter-e-do-facebook. Acesso em: 10 de abril 2015.

Da, M., Wei, W., Hu, H., and Guan, J. (2011). The application of bayesian classification theories in distance education system. International Journal of Modern Education and Computer Science, 3(4):9.

ETSI (2009). Human factors (hf); quality of experience (qoe) requirements for real-time communication services. Technical Report TR 102633.

Faulkner, L. (2003). Beyond the five-user assumption: Benefits of increased sample sizes in usability testing. Behavior Research Methods, Instruments, \& Computers, 35(3):379-383.

Ferrari, F. (2012). O estudo da entropia de transferência em sistemas dinâmicos discretos. Master's thesis, Mestrado em Ciências, Universidade Estadual de Ponta Grossa, Paraná.

Geerts, D., Katrien, M., Istvan, K., An, J., Jan, B., Wout, J., Luc, M., and Lieven, M. (2010). Linking an integrated framework with appropriate methods for measuring qoe. In Quality of Multimedia Experience (QoMEX), 2010 Second International Workshop on, pages 158-163. IEEE. 
IpsosMediact (2014). Facebook reach and usage. Estudo comissionado pelo Facebook.

ITU-T (2007). Definition of quality of experience. Technical Report SG12 109rev2, Geneva, Switzerland.

Menkovski, V., Exarchakos, G., Liotta, A., and Antonio, S. (2011). Managing quality of experience on a commercial mobile tv platform. International Journal on Advances in Telecommunications Volume 4, Number 1 \& 2, 2011.

Norman, D. (2004). Why we love (or hate) everyday things. Emotional Design.

Piccolo, L., Bonadia, G., and Tambascia, C. (2010). Aplicação de técnicas e métodos de ihc para avaliar a qoe de serviços de tv interativa. In Proceedings of the IX Symposium on Human Factors in Computing Systems, pages 225-230. Brazilian Computer Society.

Sarabando, A. (2011). Um estudo do comportamento de redes bayesianas no prognóstico da sobrevivência no cancro da próstata. Master's thesis, Faculdade de Ciências e Medicina, Universidade de Porto, Portugal.

Seruffo, M., Miranda, F., Oliveira, E., and Malcher, A. (2015). Dengue fever: Prevention through information. TVX 2015.

Soskic, N. (2014). Second screen user experience: A new digital television frontier. Information and Communication Technology, Electronics and Microelectronics (MIPRO), pages $1057-1060$.

Xenos, M. (2004). Prediction and assessment of student behaviour in open and distance education in computers using bayesian networks. Computers \& Education, 43(4):345359.

Yong, L. (2013). User experience evaluation methods for mobile devices. In Innovative Computing Technology (INTECH), 2013 Third International Conference on, pages 281-286. IEEE.

Zhou, T. (2013). The effect of flow experience on user adoption of mobile tv. Behaviour \& Information Technology, 32(3):263-272.

Zhu, Y., Heynderickx, I., and Redi, J. (2015). Understanding the role of social context and user factors in video quality of experience. Computers in Human Behavior, 49:412426. 